\title{
A Hierarchical Multiplex Structure Plus Model with Fuzzy Inference for VRSDP/MD Practical Transportation Problems
}

\author{
Xusheng WANG ${ }^{\mathrm{a}}$, Ismael Dongmo FODJO ${ }^{\mathrm{b}}$, Xiaomin $\mathrm{CHU}^{\mathrm{b}}$, Kewei $\mathrm{CHEN}^{\mathrm{b}, 1}$, \\ Fangyan DONG $^{\mathrm{b}}$ and Kaoru HIROTA ${ }^{\mathrm{c}}$ \\ a Fudan University, China \\ ${ }^{\mathrm{b}}$ Ningbo University, China \\ ${ }^{\mathrm{c}}$ Beijing Institute of Technology, China
}

\begin{abstract}
By introducing the Vehicle Routing, Scheduling \& Dispatching Problem for Multiples Depot (VRSDP/MD) and the description of formalization, it is helpful to offer a solution to solve the complex situation in practical transportation problem. In order to decrease the influence of the problem, A computing model embodying Hierarchical Multiplex Structure to take shape with an object-oriented multiple paradigms (HIMS+ model) are put forward as a proposal. And HIMS+ is divided into three layers: the first layer is the system cost adjusting area, the second layer is formation area for the system state, and that, the third layer is decision-making area for the system optimization. Two methods of meta-heuristic and fuzzy inference are proposed as an optimality calculation for HIMS+ architecture. There are two types of 24 tank lorries in the metropolis of Japan (Tokyo area), where two types of experiments are scheduled for 3-day of actual order data. Experiments results show that the HIMS+ model increase acceleration by 10 percent and decreases fast by 75 percent compared to what experts predict. The HIMS+ model has become a very reliable computing architecture for the multi objective and multi constraint optimization to real world transportation problems.
\end{abstract}

Keywords. VRSDP/MD, HIMS+ model, Intelligent Computing, Fuzzy inference.

\section{Introduction}

The VRSDP/MD problem (vehicle routing, scheduling and scheduling problem) is a significant research area developed in the last 20 years. Plenty of researchers attempt to answer basic research questions [1,2] using SA [3,4], TS [5,6], GA [7], etc. With the development of society, great changes have taken place in the modes of transportation. Centralized processing of multi-point large-scale deliveries has gradually become an important way in our life. Along with the growing for PC (personal computer) \& GIS (geographic information system), the needs to soft computing are bigger and bigger, which the practical dispatch and delivery problems can be ameliorated by the flexible

${ }^{1}$ Corresponding Author, Kewei CHEN, School of Mechanical Engineering \& Mechanics, China; Email: chenkewei@nbu.edu.cn. 
and feasible way. The VRSDP/MD problem for Multiples Depot is a general problem for real world transportation application.

In this paper, the VRSDP/MD problem is explained to model complex real-world situations in an integrated approach. For the solution of the VRSDP/MD problem, an expanded computing model with Hierarchical Multiplex Structure (HIMS+ model) is suggested. The HIMS+ model contains 3 layers: first layer, second layer and the third one. The first layer controls system costs with heuristics, the second layer adjusts the system state by heuristic and optimization methods, and Fuzzy inference is used to correct system balance at the third layer. The HIMS+ model is built as a software architecture module containing object-oriented multiple paradigms. The corresponding fuzzy inference and optimizing calculation on the basis of the heuristic algorithm is also suggested.

The experiments (two types) are verified through the order data (3 days) which is taken from a practice dispatching center in the metropolis of Japan (Tokyo area). There are 24 tank lorries (two kinds) which are used as daily dispatching for 3 depots to 6030 destinations. The delivery area within the experiment is the metropolitan area of Tokyo. By the viewpoint algorithm, the practical system application is analyzed in detail through the experimental results. Finally, this experiment results shows that the HIMS+ model is a very useful tool that is reliable, high-speed and efficient. The HIMS+ model can be built in the dispatching supporting system for the VRSDP/MD application.

The second section mainly gives the narration of the VRSDP/MD problem and the formalization by mathematical set is suggested. The third section mainly introduces the framework and computing standard of the HIMS+ model. The fourth section mainly shows experimental and appraising data by human expert contrast.

\section{Definition \& Formulation to VRSDP/MD Problem}

In this section, the daily delivery activities of VRSDP/MD problem in real life are mainly introduced. The VRSDP/MD problem is a synthesis problem which contains three sub-problems in life, like routing problem, scheduling problem and dispatching problem. The VRSDP/MD problem has many practical applications, such as food and beverage delivery by vehicle in running 24 hours stores, oil transportation by tanker truck in gas stations, etc.

\subsection{The Concept of VRSDP/MD}

In this subsection, the actual daily delivery issues are based on VRSDP/MD problem (figure 1). Depots D is the distribution center used to store all goods and has several vehicles $\left\{V_{l}\right\}$ of different sizes for distribution. When: (1) Select $N$ users $\left\{U_{n}\right\}$ from the order consumers, and use these users to obtain $\mathrm{M}$ orders $\left\{O_{m}\right\}$ for certain requirements in a certain time window. (2) Each user 's are different from each other in distribution cars parking space, business hours and so on. Delivery schedules must be made for all the different models of $\mathrm{R}$ cars on these $\mathrm{M}$ orders until the next day. The problem is how to make optimal route and efficient scheduling for all the distribution work, and make proper scheduling for various types of vehicles under certain constraints. 


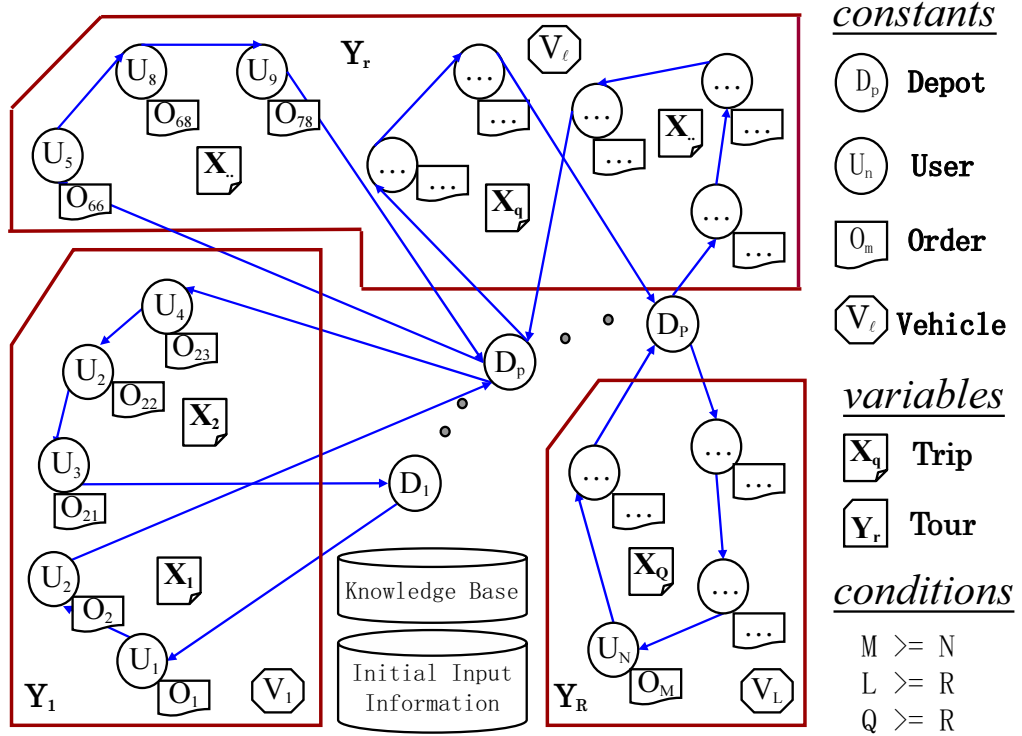

Figure 1. The concept of VRSDP/MD

\subsection{Fixed Values and Variables}

Table 1. Fixed values in VRSDP/MD.

\begin{tabular}{cccc}
\hline Item & Symbol & Number & Universal Set \\
\hline Depot & $\mathrm{D}_{\mathrm{p}}$ & $\mathrm{P}$ & Set $\mathrm{D}=\left\{\mathrm{D}_{1}, \ldots, \mathrm{D}_{\mathrm{p}}, \ldots, \mathrm{D}_{\mathrm{P}}\right\}$ \\
User & $\mathrm{U}_{\mathrm{n}}$ & $\mathrm{N}$ & $\mathrm{Set} \mathrm{U}=\left\{\mathrm{U}_{1}, \ldots, \mathrm{U}_{\mathrm{n}}, \ldots, \mathrm{U}_{\mathrm{N}}\right\}$ \\
Order & $\mathrm{O}_{\mathrm{m}}$ & $\mathrm{M}$ & Set $\mathrm{O}=\left\{\mathrm{O}_{1}, \ldots, \mathrm{O}_{\mathrm{m}}, \ldots, \mathrm{O}_{\mathrm{M}}\right\}$ \\
Vehicle & $\mathrm{V}_{l}$ & $\mathrm{~L}$ & Set $\mathrm{V}=\left\{\mathrm{V}_{1}, \ldots, \mathrm{V}_{l}, \ldots, \mathrm{V}_{\mathrm{L}}\right\}$ \\
\hline
\end{tabular}

Table 1 expresses Fixed values in VRSDP/MD. Each Fixed value is defined in detail as follows:

$$
D_{p}=\left(\left[B t_{p}, E t_{p}\right]\right)
$$

Both $B t_{p}$ (Starting Time for Depot) and $E t_{p}$ (Ending Time for Depot) are measured in minutes and integer values. The integer range between $B t_{p}$ and $E t_{p}$ is the time window for the loading works.

$$
U_{n}=\left(\left[B t_{n}, E t_{n}\right], S U_{n}\right)
$$


The integer interval between $B t_{n}$ and $E t_{n}$ is the constraint of the business time, and $S U_{n}$ shows maximum size of the vehicle which $U_{n}$ can be entered.

$$
O_{m}=\left(C_{m},\left[B t_{m}, E t_{m}\right], U_{n}^{m}\right), C_{m} \leq S U_{n}^{m}
$$

The $C_{m}$ Indicates the user $\left(U_{n}^{m}\right)$ order capacity, and the integer interval between $B t_{m}$ and $E t_{m}$ is the required delivery tine window.

$$
V_{l}=\left(S V_{l}, R W t_{l}, E W t_{l}, D_{p}^{l}\right)
$$

In the above, $S V_{l}$ represents the maximum capacity that the vehicle can carry, $R W t_{l}$ represents the constraint working time until when $V_{l}$ must work full, and $E W t_{l}$ is the max extended working time until when $V_{l}$ can work more. $D_{p}^{l}$ is the garage depot that belongs of $V_{l}$.

The $S U_{n}, C_{m}$, and $S V_{l}$ have integer values of the same measure, the weight of solid or the volume of liquid. The $C_{m}$ can be an optional integer value, while the $S U_{n}$ and $S V_{l}$ are some fixed integer values corresponding to the types of vehicles.

Table 2. Variables defined in VRSDP/MD.

\begin{tabular}{cccc}
\hline Item & Symbol & Number & Universal Set \\
\hline Trips & $\mathrm{X}_{\mathrm{q}}$ & $\mathrm{Q}$ & Set $\mathrm{X}=\left\{\mathrm{X}_{1}, \ldots, \mathrm{X}_{\mathrm{q}}, \ldots, \mathrm{X}_{\mathrm{Q}}\right\}$ \\
Tours & $\mathrm{Y}_{\mathrm{r}}$ & $\mathrm{R}$ & Set $\mathrm{Y}=\left\{\mathrm{Y}_{1}, \ldots, \mathrm{Y}_{\mathrm{r}}, \ldots, \mathrm{Y}_{\mathrm{R}}\right\}$ \\
\hline
\end{tabular}

Table 2 expresses the variables for trips and tours in VRSDP/MD. These variables represent the solution of the VRSDP/MD problem. Each variable is defined in detail as follows:

$$
\begin{gathered}
J=\left\{J_{1}, \ldots, J_{m}, \ldots, J_{M}\right\}, \quad J_{m}=\left(O_{m}, U_{n}^{m}\right) ; \\
X_{q}=\left(Y_{r}^{q}, k_{q},\left[D_{L}^{q}, J_{1}^{q}, \ldots, J_{i}^{q}, \ldots, J_{K_{q}}^{q}, D_{R}^{q}\right]\right), \\
J_{i}^{q} \in J, \quad K_{q} \geq 1, \quad k_{q} \in\left\{1,2, . ., K_{r}\right\}, \\
Y_{r}=\left(\left[X_{1}^{r}, \ldots, X_{j}^{r}, \ldots, X_{K_{r}}^{r}\right], V_{l}^{r}\right), \\
K_{r} \geq 1, \quad X_{j}^{r} \in X ; \\
D_{R}\left(\in X_{1}^{r}\right)=D_{L}\left(\in X_{2}^{r}\right), \ldots, D_{R}\left(\in X_{K_{r}-1}^{r}\right)=D_{L}\left(\in X_{K_{r}}^{r}\right), \\
D_{L}\left(\in X_{1}^{r}\right)=D_{R}\left(\in X_{K_{r}}^{r}\right)=D_{p}^{l}
\end{gathered}
$$

$J_{i}^{q}$ is the job i in trip $X_{q}$ with order/user pair information (the total number is $K_{q}$ ). $D_{L}^{q}$ means the loading depot and $D_{R}^{q}$ means the rally depot. $Y_{r}^{q}$ is the tour to which $X_{q}$ belongs, $X_{q}$ represents an index number of $X_{q}$ in the tour $Y_{r}^{q}$, and $K_{r}^{q}$ is the total 
number of trips in the tour $Y_{r}^{q}$. Formula 7 means the vehicle tour carried out by $V_{r}$. Where, $\left[X_{1}^{r}, \ldots, X_{j}^{r}, \ldots, X_{K_{r}}^{r}\right]$ are the trips sequence within $Y_{r}$. The constraining factors for $D_{p}^{\prime}=D_{L}\left(\in X_{1}^{r}\right)=D_{R}\left(\in X_{K_{r}}^{r}\right)$ is start or end condition to cars and $D_{R}\left(\in X_{1}^{r}\right)=D_{L}\left(\in X_{2}^{r}\right)$ is middle loading condition.

The constant information narrated above is given in a priori, so that the solution delegated by $\{X\}$ and $\{Y\}$ from some initial information can be computed on account of the constraint conditions and the constant information, which talk over the next subsection.

\subsection{Constraint Condition}

Some constraints are imposed in VRSDP/MD problem.

(1). Vehicle Capacity restriction

$$
\begin{gathered}
C_{\text {cnst }}^{q}= \begin{cases}\text { if } S V_{l}^{r} \geq \sum_{j=1}^{K_{q}} C_{j}^{q} & 0 \\
\text { else } & 1\end{cases} \\
C_{\text {cnst }}=\sum_{q=1}^{Q} C_{\text {cnst }}^{q}=0
\end{gathered}
$$

Formulas 8 and 9 enunciate the constraint conditions of vehicles, i.e., the total orders capacity within the trip can't be exceeded the corresponding vehicles capacity. Here, $C_{j}^{q}$ is the quantity of the order $\mathrm{j}$ and $S V_{l}^{r}$ is the vehicle volume for the trip $X_{q}$. (2). application condition

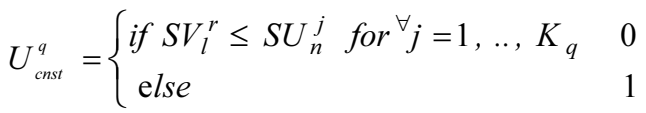

$$
\begin{aligned}
& U_{\text {cnst }}=\sum_{q=1}^{Q} U_{c n s t}^{q}=0
\end{aligned}
$$

The constraint expressed in formulas 10 and 11 is that the size of the vehicle must be equal to or less than the parking spaces of all users of the trip it performs. Where, $S U_{n}^{j}$ is the parking space of the user order $\mathrm{j}$ in the trip $X_{q}$.

(3). Condition of Depot

$$
\begin{gathered}
D_{\text {cnst }}^{q}= \begin{cases}\text { if } B t_{p} \leq A t_{p}^{q} \leq E t_{p} & 0 \\
\text { else } & 1\end{cases} \\
D_{\text {cnst }}=\sum_{q=1}^{Q} D_{\text {cnst }}^{q}=0
\end{gathered}
$$


Formulas 12 and 13 show all loading work is bound to be carried out when the depot is working.

Here $A t_{P}^{q}$ is the vehicle arriving time with the trip $X_{q}$ reach to $D_{q}$ depot for the loading.

(4). Dispatching Condition

$$
R \leq Q ; \quad R \leq L ; \quad N \leq M
$$

The constraints condition in formula 14 show:

I). The user is bound to have at least one order per day;

II). Tours $(R)$ size must be equal to or less than the total vehicles $(L)$ size;

III). One trip is bound to make per day.

\subsection{Evaluation Functions to VRSDP/MD Problem}

This section defines VRSDP/MD problems related to some evaluation functions, where the mark "... $\Rightarrow \ldots$ " indicates the item state on the right is eager to reach the values described on the left.

the right item state is eager to reach the value of the left description.

(1). Total Running Cost

$$
\min \Rightarrow T_{\cos t}=\sum_{q=1}^{Q} T_{\cos t}^{q}
$$

The formula 15 describes the total running cost. Formula 16 calculates the operation cost (distance, time, etc.) of $X_{q}$. The $\left.t_{a, b}^{q}\left(a, b \in\left\{U_{n}\right\} U_{\{} D_{q}\right\}\right)$ is the moving cost among $a$ and $b$, the $t_{\text {load }}^{q}$ is the loading cost, the $t_{\text {unload, } U_{i}}^{q}$ or the $t_{\text {wait, } U_{i}}^{q}$ are the unloading time and the waiting time at the user $U_{i}$, separately. Here, $U_{i}$ indicates the user having the order i $\left(O_{i}^{q}\right)$ within trip $X_{q}$. This time information can be computed on the basis of the database consisting of the depot, the user $\&$ the road network information.

$$
\begin{array}{r}
T_{\cos t}^{q}=t_{D L, U_{1}}^{q}+\sum_{i=1}^{K_{q}-1} t_{U_{i}, U_{i+1}}^{q}+t_{U_{K_{q}}, D R}^{q}+ \\
t_{\text {load }}^{q}+\sum_{i=1}^{K_{q}}\left(t_{\text {unload }, U_{i}}^{q}+t_{\text {wait }, U_{i}}^{q}\right)
\end{array}
$$

(2). Average Capacity of Loading

$$
\begin{gathered}
\max \Rightarrow C_{\cos t}=\frac{1}{Q} \sum_{q=1}^{Q} C_{\cos t}^{q} \\
C_{\text {cost } t_{\in[0,1]}}^{q}=\left(\sum_{j=1}^{K_{q}} C_{j}^{q}\right) / S V_{l}^{r}
\end{gathered}
$$


Formulas 17 and Formulas 18 show the average carrying capacity rate for all trips. High values of $C_{\text {cost }}$ are always desirable in order to improve transportation efficiency. Where, $C_{j}^{q}$ is the order $\mathrm{j} O_{j}^{q}$ capacity within $X_{q}, S V_{l}^{r}$ is the vehicle capacity carrying out tour $Y_{r}^{q}$.

(3). Vehicles Working Balance

$$
\begin{aligned}
\min \Rightarrow B_{\cos t} & =\frac{1}{R} \sum_{r=1}^{R}\left|B_{\cos t}^{r}-B_{\cos t}^{\text {mean }}\right| \\
B_{\cos t}^{r} & =\sum_{i=1}^{K_{r}} T_{\cos t}^{i, r} \\
B_{\cos t}^{\text {mean }} & =\frac{1}{R} \sum_{r=1}^{R} B_{\cos t}^{r}
\end{aligned}
$$

The vehicles working balance are also conditioned, which are very influential for fair on the labour condition for drivers at work. The work balance of the vehicle is also taken into account, The $B_{\text {cost }}^{r}$ is the running cost for the tour $Y_{r}$, and the $T_{c o s t}^{i, r}$ is the running cost of the trip I ( $X_{i}^{r}$ ) in tour $Y_{r}$ and are defined by the Formula 16. Since the work balance is the dynamic state of the vehicle at work, the average in Formula 19,21 can be used to calculate the vehicle $\mathrm{R}$ in use.

(4). Vehicles Working Capacity

$$
\max \Rightarrow V_{\text {cost }}=L-R
$$

In order to reduce the running cost, according to the number of orders, to use a suitable number of vehicles is better. This Formula 22 means the working capacity.

(5). Fuzzy measures one (Fuzzy measures for time constraint)

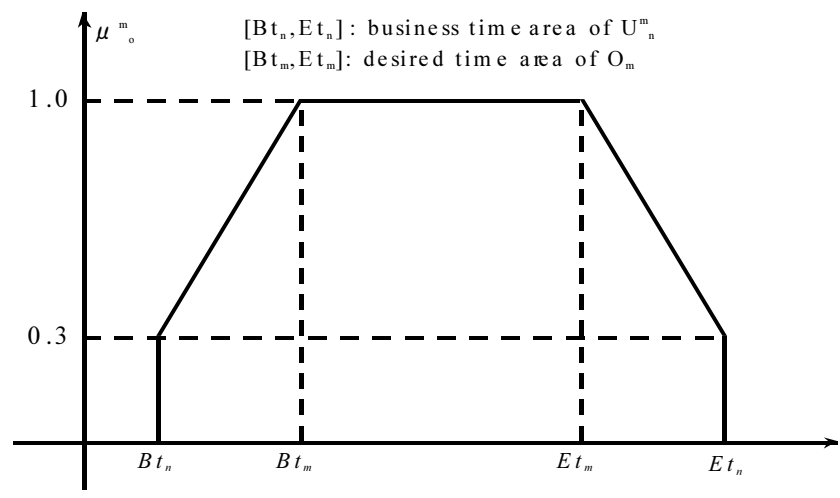

Figure 2. Fuzzy measures for time constraint 


$$
\begin{gathered}
\max \Rightarrow S_{T}=\frac{1}{Q} \sum_{q=1}^{Q} S_{T}^{q} \\
S_{T}^{q}=\frac{1}{K_{q}} \sum_{j=1}^{K_{q}} \mu_{o}^{j}
\end{gathered}
$$

User orders usually have a specified delivery time that must be met as far as possible. Suppose that the $\mu_{O}^{m}$ is user $U_{n}^{m}$ 's satisfaction with the delivery time of $O_{m}$, which be calculated by using the fuzzy membership function in Figure 2.

(6). Fuzzy measures two (Fuzzy measure for work load)

$$
\max \Rightarrow S_{V}=\frac{1}{L} \sum_{l=1}^{L} \mu_{V}^{l}
$$

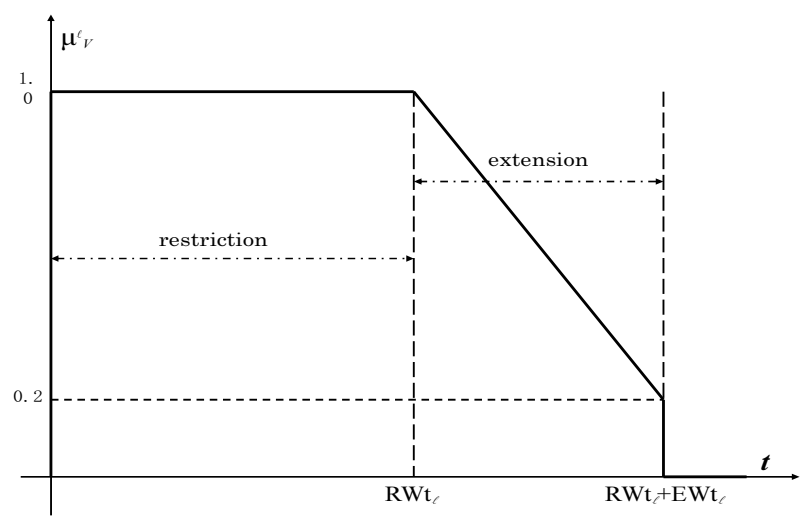

Figure 3. Fuzzy measures for work load.

In modern society, the labour limit of drivers must be conditioned. As one vehicle corresponds to one driver for the VRSDP/MD problem, the constraint about driver working is considered by the vehicle corresponding with the attributes (EWt or RWt). the limit about vehicle working is called the fuzzy measures two, which is defined as the Formula 25. In Figure 3, The driver's operating state of vehicle $V$ is described as the fuzzy membership function $\mu_{V}^{l}$. Where a car is parked out of use, it should be normal (not affected by $S_{v}$ ) and safe $\left(\mu_{V}^{l}=1\right)$. Even though this constraint is not as important as fuzzy measures one, it can give consideration to the trade-off between the initial value of the limited working hours and the number of vehicles used.

\section{HIMS+: Expanded Calculation Model}

In order to solve the VRSDP/SD problem [8], Hierarchical Multiplex Structure which is a calculation model has been proposed. In this paper, A model that called the HIMS+ is proposed, basing on HIMS model. And HIMS+ model is an expanded than HIMS 
model. The HIMS+ model has three layers like HIMS. And HIMS+ is divided into three layers: the first layer is the system cost adjusting area, the second layer is formation area for the system state, and that, the third layer is decision-making area for the system optimization. The HIMS+ model can process multiple depots for VRSDP/MD problem that is different the HIMS model, such as control and modify the relationship among trips, tours, and depots actively by changing loading stations (figure 4).

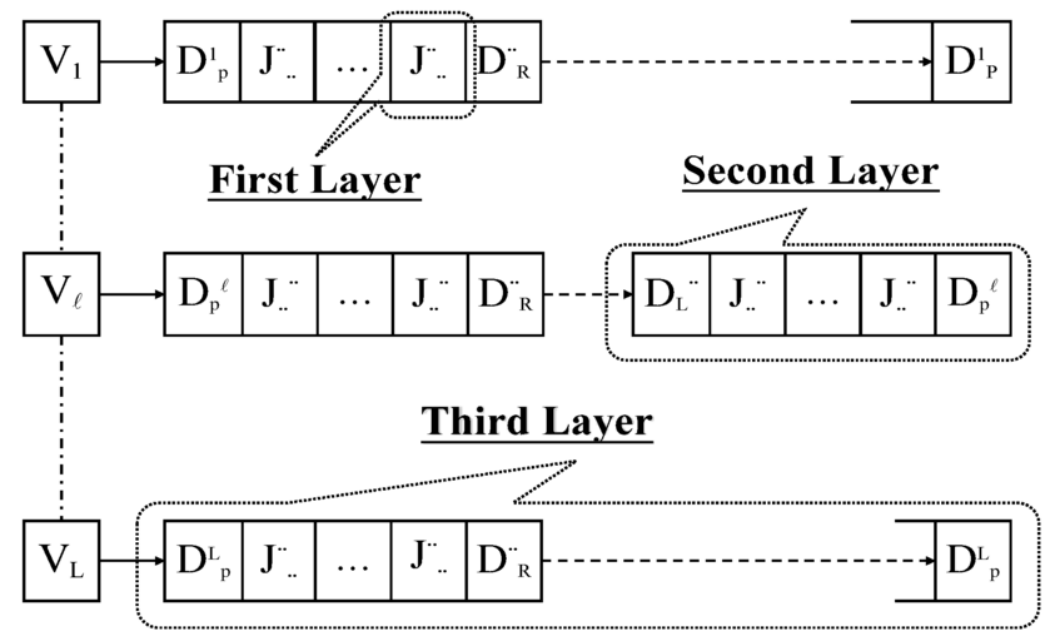

Figure 4. The definition of the HIMS+ Model.

In order to deal with the VRSDP/MD problem, the HIMS+ model also mimics the hierarchical computing and reasoning characteristics of human experts and divides the VRSDP/MD problem into sub-problems. Then, sub-problems are solved at different levels.

In this section, this paper introduces the HIMS+ model's operation strategy, some measures and choice function, and an algorithm for optimization.

\subsection{First Layer Strategy}

The goals in the First layer are:

(1). Getting minimum the working costs

(2). Making the carrying capacity stronger.

(3). Enhancing customer satisfaction degree in Fuzzy measures two. 
${ }^{\exists} X_{\mathrm{a}}=\left(\left[D_{L}^{\mathrm{a}}, J_{1}^{\mathrm{a}}, . ., J_{i}^{\mathrm{a}}, . ., J_{K a}^{\mathrm{a}}, D_{R}^{\mathrm{a}}\right], Y_{r}^{\mathrm{a}}, k_{\mathrm{a}}\right)$, where, $1 \leq \mathrm{a} \leq Q$;

${ }^{\exists} X_{b}=\left(\left[D_{L}^{b}, J_{1}^{b}, . ., J_{j}^{b}, . ., J_{K b}^{b}, D_{R}^{b}\right], Y_{r}^{b}, k_{b}\right)$, where, $1 \leq b \leq Q$;

(1) . Order Move Operation

$$
\begin{aligned}
& X_{a^{\prime}}=\left(\left[D_{L}^{\mathrm{a}}, J_{1}^{\mathrm{a}}, . ., J_{\mathrm{i}}^{\mathrm{a}}, J_{\mathrm{j}}^{\mathrm{b}}, J_{i+1}^{\mathrm{a}}, . ., J_{K a^{\prime}}^{\mathrm{a}}, D_{R}^{\mathrm{a}}\right], Y_{r}^{\mathrm{a}}, k_{\mathrm{a}}\right), \\
& X_{b^{\prime}}=\left(\left[D_{L}^{b}, J_{1}^{b}, . ., J_{j-1}^{b}, J_{j+1}^{b}, \ldots, J_{K b}^{b}, D_{R}^{b}\right], Y_{r}^{b}, k_{b}\right) ;
\end{aligned}
$$

(2). Order Exchange Operation

$$
\begin{aligned}
& X_{a^{\prime}}=\left(\left[D_{L}^{\mathrm{a}}, J_{1}^{\mathrm{a}}, . ., J_{\mathrm{i}-1}{ }^{\mathrm{a}}, J_{j}{ }_{j}^{\mathrm{b}}, J_{i+1}{ }^{\mathrm{a}}, . ., J_{K \mathrm{a}}^{\mathrm{a}}, D_{R}^{a}\right], Y_{r}^{a}, k_{\mathrm{a}}\right), \\
& X_{b^{\prime}}=\left(\left[D_{L}^{b}, J_{1}^{b}, . ., J_{j-1}^{b}, J_{i}^{\mathrm{a}}, J_{j+1}^{b}, . ., J_{K b}^{b}, D_{R}^{b}\right], Y_{r}^{b}, k_{b}\right) ;
\end{aligned}
$$

To Satisfy :

I. $\operatorname{TSP}\left(X_{\mathrm{a}^{\prime}}\right), \operatorname{TSP}\left(X_{b^{\prime}}\right)$,

II. $T_{\text {cost }}^{\mathrm{a}^{\prime}}+T_{\text {cost }}^{\mathrm{b}^{\prime}} \leq T_{\text {cost }}^{\mathrm{a}}+T_{\text {cost }}^{\mathrm{b}}$;

Figure 5. Orders execution conditions in First Layer.

At the First Layer, this adjustment of order is thought to be analogous to the movement of atoms. The Exchange/Move operations can be performed frequently to build trips as molecules in the optimizing state (lowest running cost). The operational strategy of the First Layer (figure 5) is introduced as follows:

First of all, the original state is $M$ trips, and each trip has one order, and operation one and two are performed multiple times in figure 5 . After each operation, the optimal route $\operatorname{TSP}\left(X_{q}\right)$ is computed for each updated trip $\left(X_{a}\right.$, or $X_{b}$, by changing its job's order, and a feasible solution to reduce the operation cost is found for TS (Tabu Search) [9] and other heuristic methods that is used to apply instruction Exchange/Move operation and to find the shortest path $\operatorname{TSP}\left(X_{q}\right)$ with SA (Simulated Annealing [10]).

\subsection{Second Layer Strategy}

The Second Layer operational goals are:

(1). Making the work balance more stable.

(2). Making the loading capacity stronger.

(3). Enhancing customer satisfaction degree in Fuzzy measures one.

(4). Reducing the operating cost. 
At the Second Layer, the trip is considered to be a Molecule. The operation of Exchange/Move can be performed continually to build the most suitable individuals (tours). The cost of linking molecules (trips) can be reduced by adjusting (Middle Depot Change) some of the elements (depots). The operational strategy of the Second Layer (figure 6,7) is introduced as follows:

As in the original state, $L$ tours (equal to the number of working vehicles) is accomplished by building the trips at the first layer. The stroke Exchange/Move operation is then performed between the tours (figure 6). After per operation, the updated tour $\left(Y_{a}\right.$, or $Y_{b}$ ) will be adjusted to rationalize the works schedule of trips. The updated tour ( $\mathrm{Ya}$ 'or $\mathrm{Yb}$ ') will be adjusted to make a better work plan of trips. In order to reduce running costs, the intermediate depot interchange operation (figure 7) is carried out during the trip.

In the figure $6,\left|B_{\text {cost }}^{a}-B_{\text {cost }}^{b}\right|$ and $\left|B_{\text {cost }}^{a^{\prime}}-B_{\text {cost }}^{b^{\prime}}\right|$ are the difference between a and b tours operating costs before and after the Exchange/Move operation. $C_{\text {cost }}^{Y_{a^{\prime}}}$ and $S_{T}^{Y_{a^{\prime}}}$ are the average carrying capacity and service time of each trip in the updated tourism $Y_{a}$.

$$
\begin{aligned}
& { }^{\exists} Y_{a}=\left(V_{l}^{\mathrm{a}},\left[X_{1}^{\mathrm{a}}, . ., X_{i}^{\mathrm{a}}, . ., X_{I}^{\mathrm{a}}\right]\right), \quad 1 \leq \mathrm{a} \leq R ; \\
& { }^{\exists} Y_{b}=\left(V_{l}^{b},\left[X_{1}^{b}, . ., X_{j}^{b}, . ., X_{J}^{b}\right]\right), \quad 1 \leq b \leq R \text {; } \\
& \text { (1) . Trip Move Operation } \\
& Y_{a^{\prime}}=\left(V_{1}^{\mathrm{a}},\left[X_{1}^{\mathrm{a}}, \ldots, X_{i}^{\mathrm{a}}, X_{j}^{\mathrm{b}}, X_{i+1}^{\mathrm{a}}, . ., X_{\mathrm{I}}^{\mathrm{a}}\right]\right), \\
& Y_{b},=\left(V_{l}^{b},\left[X_{1}^{b}, \ldots, X_{j-1}^{b}, X_{j+1}^{b}, \ldots, X_{J}^{b}\right]\right) \text {; } \\
& \text { (2). Trips Exchange Operation } \\
& Y_{a^{\prime}}=\left(V_{l}^{\mathrm{a}},\left[X_{1}^{\mathrm{a}}, \ldots, X_{i-1}^{\mathrm{a}}, X_{j}^{b}, X_{i+1}^{\mathrm{a}}, . ., X_{\mathrm{I}}^{\mathrm{a}}\right]\right), \\
& Y_{b},=\left(V_{l}^{b},\left[X_{1}^{b}, . ., \quad X_{j-1}^{b}, X_{i}^{a}, X_{j+1}^{b}, . ., X_{J}^{b}\right]\right) \text {; }
\end{aligned}
$$

Satisfied by :

$$
\begin{array}{ll}
\text { I. } & \left|B_{\text {cost }}^{\mathrm{a}^{\prime^{\prime}}}-B_{\text {cost }}^{\mathrm{b}^{\prime}}\right|<\left|B_{\text {cost }}^{\mathrm{a}}-B_{\text {cost }}^{\mathrm{b}}\right| \\
\text { II } . & C_{\text {cost }}^{\mathrm{Y}^{\mathbf{a}^{\prime}}}+C_{\text {cost }}^{\mathrm{Y}_{\mathrm{b}^{\prime}}} \geq C_{\text {cost }}^{\mathrm{Ya}^{\mathrm{a}}}+C_{\text {cost }}^{\mathrm{Y}_{\mathrm{b}}} \\
& C_{\text {cost }}^{\mathrm{Ya}_{\mathrm{a}}}=\sum_{q \in Q^{\prime}} C_{\cos t}^{q}, Q^{\prime}=\left\{q \in\{1,2, \ldots, Q\} \mid X_{q} \in Y_{a}\right\} \\
\text { III } . & S_{T}^{\mathrm{Y}_{\mathrm{a}^{\prime}}}+S_{T}^{\mathrm{Y}_{\mathrm{b}^{\prime}}} \geq S_{T}^{\mathrm{Y}_{\mathrm{a}}}+S_{T}^{\mathrm{Y}_{\mathrm{b}}} \\
& S_{T}^{Y_{a}}=\sum_{q \in Q^{\prime}} S_{T}^{q}, \quad Q^{\prime}=\left\{q \in\{1,2, \ldots, Q\} \mid X_{q} \in Y_{a}\right\}
\end{array}
$$

Figure 6. Trips execution conditions in Second Layer. 


$$
\begin{aligned}
{ }^{\exists} J^{\mathrm{i}} & =\left[J_{1}^{\mathrm{i}}, J_{2}^{\mathrm{i}}, \ldots, J_{K i}^{\mathrm{i}}\right], \\
{ }^{\exists} Y_{a} & =\left(V_{l}^{a},\left[. ., X_{\mathrm{i}-1}:\left(D_{L}^{\mathrm{i}-1}, J^{\mathrm{i}-1}, D_{R}^{i-1}\right), X_{i}:\left(D_{L}^{\mathrm{i}}, J^{\mathrm{i}}, D_{R}^{i}\right), . .\right]\right), \\
& { }^{\forall}, \quad D_{R}^{i-1}=D_{L}^{i}, \quad 1 \leq a \leq R, 2 \leq i \leq K_{a} ;
\end{aligned}
$$

(1) . Middle Depot Change Operation

$$
\begin{aligned}
& Y_{a^{\prime}}=\left(V_{l}^{a},\left[. ., X_{\mathrm{i}-1}:\left(D_{L}^{\mathrm{i}-1}, J^{\mathrm{i}-1}, D_{p}\right), X_{\mathrm{i}}:\left(D_{p}, J^{\mathrm{i}}, D_{R}^{\mathrm{i}}\right), . .\right]\right) ; \\
& \qquad D_{p} \neq D_{L}^{\mathrm{i}} ; \\
& \text { Satisfied by : } \quad T_{\text {cost }}^{\mathrm{Y}_{a^{\mathrm{i}}}}<T_{\text {cost }}^{\mathrm{Y}_{\mathrm{a}}}
\end{aligned}
$$$$
T_{\cos t}^{Y_{q}}=\sum_{q \in Q^{\prime}} T_{\cos t}^{q} \text {, where, } Q^{\prime}=\{\mathrm{q}=1,2,3, \ldots, Q \mid X \mathrm{q} \in \mathrm{Ya}\}
$$

Figure 7. Depot execution conditions in Second Layer.

The $T_{\text {cost }}^{Y_{a^{\prime}}}$ is the running cost of the trips in the updated tour $Y_{a}$, as shown in figure 7.

TS (Tabu Search) is also used to apply similar trip or warehouse Exchange/Move operations in the First layer. In addition, GS (Global Search) is implemented through a recursive process to rescheduling the trips in the updated trip to enhancing Fuzzy measures one. Because $\mathrm{Kr} \leqq 6$ is satisfied in all instances, GS is able to find the best order to quickly update the trip.

\subsection{The Third Layer: Fuzzy Inference}

The goals in the Third layer are as follows.

(1) Enhancing the vehicles working balance.

(2) Allocation the vehicles appropriate number.

Transform multiple knowledge bases into fuzzy membership functions, as shown in Figure 8 and Figure 9. Where, $T_{R}^{A}$ is the total time limit for class A tours by vehicles of the same size and $T_{\cos t}^{A}$ is the total working time of all vehicles of A type. $\mathrm{N}\left(\mathrm{X}_{\mathrm{B}}\right)$ shows the amount of the total trips made by the $\mathrm{B}$ type vehicles, and $\mathrm{N}\left(\mathrm{Y}_{\mathrm{A}}\right)$ shows the amount of the total tours made by the $A$ type vehicles.

$T_{R}^{A}$ is the total time limit for class A tours by vehicles of the same size.

Figure 8 demonstrates the trip balancing operation between different types of vehicles. The datum lines of a trip balance, the $\mu_{L}$ (lower boundary) and the $\mu_{U}$ (upper boundary) are given. The $\mu_{T}$ (the current value of trip balance) can be used to decide whether to adjust the trip balance of the Third layer. 


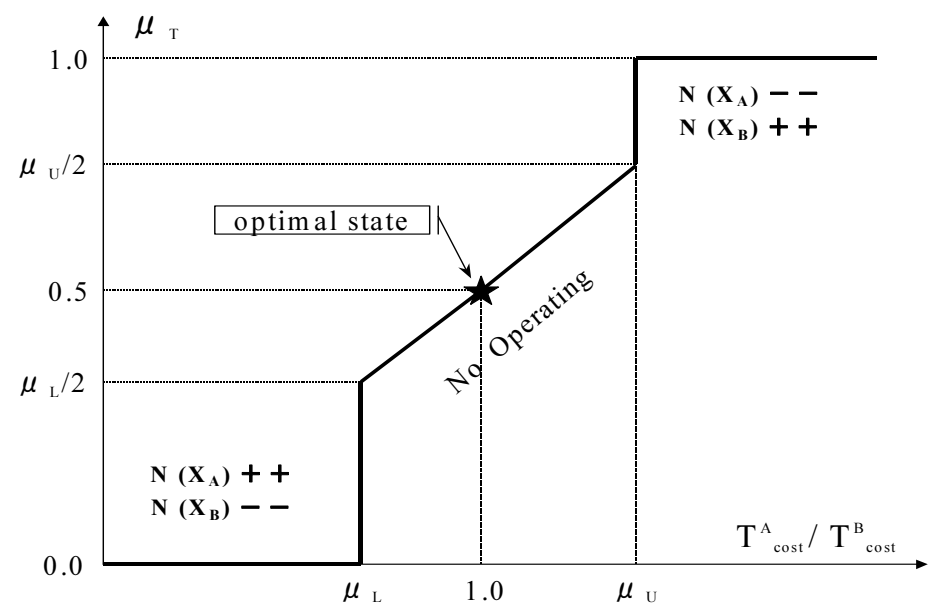

Figure 8. Trip adjustment by fuzzy measure.

Figure 9 demonstrates the vehicle balancing operation for the same type of vehicle. The vehicle balance reference lines $\lambda_{L}$ (lower bound) and $\lambda_{L}$ (upper bound) are given.

$\lambda_{v}$ (Current vehicle balance value) is used to determine whether to adjust the vehicle balance of the Third layer.

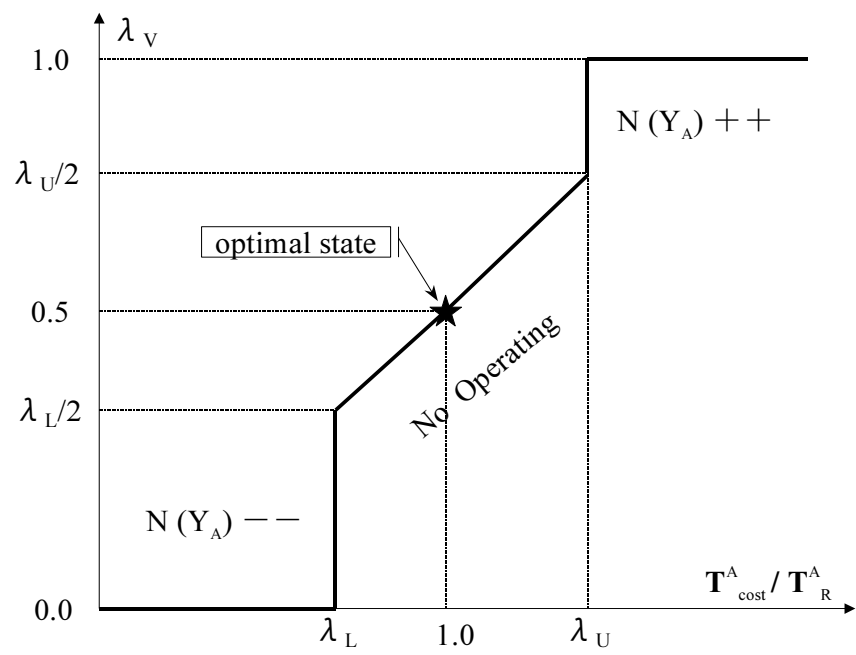

Figure 9. Vehicle adjustment by fuzzy measure.

In the third layer, two fuzzy membership functions can not only adjust the relationship between trips and tours, but also reasonably arrange different types of vehicles. 


\subsection{Measurement and Choices}

This section defines standard for measuring temporary states and for selecting locally optimal states of $\{\mathrm{X}\}$ and $\{\mathrm{Y}\}$.

\subsubsection{Mass Measurement.}

(1) Measuring of total work costs

$$
g_{1}\left(X^{k}\right)_{\in[0,1]}=1-T_{\text {cost }}^{[k]} / T_{\text {cost }}^{[0]}
$$

(2) Measuring of average carrying capacity

$$
g_{2}\left(X^{k}\right)_{\in[0,1]}=C_{\cos t}^{[k]}
$$

(3) Measuring of working balance

$$
g_{3}\left(Y^{k}\right)_{\in[0,1]}=1-B_{\text {cost }}^{[k]} / B_{\text {cost }}^{[0]}
$$

(4) Measuring of working capacity

$$
g_{4}\left(Y^{k}\right)_{\in[0,1]}=1-\left(1+V_{\text {cost }}^{[k]}\right)^{-1}
$$

(5) Measuring of Fuzzy measures one

$$
g_{5}\left(X^{k}\right)_{\in[0,1]}=S_{T}^{[k]}
$$

(6) Measuring of Fuzzy measures two

$$
g_{6}\left(Y^{k}\right)_{\in[0,1]}=S_{V}^{[k]}
$$

Formulas $26 \sim 31$ introduce the measurement standard of each objective state, in which superscript [0] represents the initial value of each objective state, and superscript [k] represents the state value that generates the temporary state. The value of $g_{x}$ is directly proportional to the objective state. The higher the $g_{x}$ value, the better the objective state.

$$
g\left(Y^{k}, X^{k}\right)=\sum_{i=1}^{6} \rho_{i} g_{i}\left(Y^{k} \mid X^{k}\right) ; \rho_{i} \in R^{+}
$$

Formula 32 describes the general measuring formula for all objective items, where $\rho_{i}$ is the weighting coefficient, representing the importance degree of per objective item. 


\subsubsection{Choice Criteria for Local Optimum States.}

In Formula 33, the local optimal solution of VRSDP/MD problem can be found by defining the fitness value of HIMS + model. Formulas 34 indicates whether or not to collect the better information of $\{X\}$ and $\{Y\}$, where the minimum value of each measurement results is denoted by $a_{i}(i=1 \sim 6)$.

$$
\begin{aligned}
& \operatorname{eval}(k)=\frac{g\left(Y^{0}, X^{0}\right)-g\left(Y^{k}, X^{k}\right)}{g\left(Y^{0}, X^{0}\right)-g\left(Y^{*}, X^{*}\right)} \\
& g\left(Y^{*}, X^{*}\right)= \begin{cases}\text { if eval }(k)>1 & g\left(Y^{k}, X^{k}\right) \\
\text { else } & g\left(Y^{*}, X^{*}\right)\end{cases} \\
& \text { s.t. } g_{i} \geq a_{i} \quad(i=1 \sim 6)
\end{aligned}
$$

\subsection{Realization of HIMS+ model}

The data architecture of HIMS+ model for application-oriented programming is proposed. This section also introduces optimization algorithms using multiple heuristics.

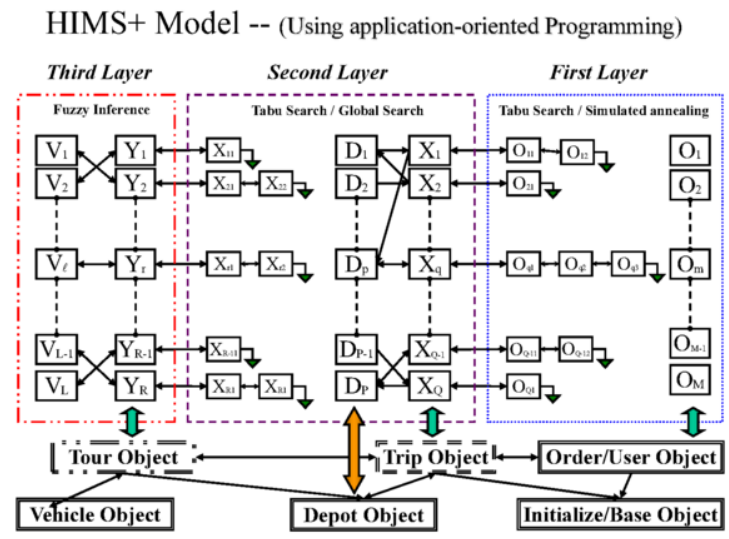

Figure 10. The HIMS+ Model Software architecture.

\subsubsection{HIMS + Model Data Architecture}

The figure 10 shows the implementation of applying application-oriented programming techniques to the HIMS+ model. The key elements in this model have hidden link relationships between object classes and double-linked Pointers, so that information can be obtained directly. The processes in 3 Layers (First, Second, and Third) are executed by meta-programming and fuzzy inference. 
By using hierarchical and multiplex data architectures, the data architecture of HIMS+ model can decompose multi-object synthesis problems such as VRSDP/MD into sub-problems. So, the combination of all elements can be searched though metaprogramming. At different levels, fuzzy inference programming is used to adjust the relationship between various elements.

\subsubsection{The Algorithm of Optimization for HIMS+ Model.}

In figure 11, The algorithm of optimization in HIMS+ model data architecture is depicted.

In the first layer, through the heuristic method, TS (Tabu Search), uses the information of $\{\mathrm{O}\},\{\mathrm{U}\}$, and $\{\mathrm{D}\}$ to construct $\{\mathrm{X}\}$, which is used to deal with routing problems. In the second layer, according to the information of $\{\mathrm{X}\}$ and $\{\mathrm{V}\}$, finding $\{\mathrm{Y}\}$ by TS can be used to solve the scheduling problems.

According to the relationship among $\{\mathrm{X}\},\{\mathrm{Y}\}$, and $\{\mathrm{V}\}$, trip adjusting $\mu_{T}$ (In multiple types) can be got (See figure 8 for details). While $\mu_{T} \geq \mu_{U}$, The trip of type $A$ will be shifted or divided into the trips of type $B$ and while $\mu_{T} \leq \mu_{L}$ vice versa. Similarly, $\lambda_{V}$, the balance of the same type of vehicle can be gained (See figure 9). While $\lambda_{T} \geq \lambda_{U}$, in order to reduce too much work, A new vehicle of type A will be reassigned and while $\lambda_{T} \leq \lambda_{L}$, The vehicle will be removed from tour and the relevant trip from that tour will be moved to another tour. In the Third Layer, the scheduling problem is dealt with by fuzzy reasoning comprehensive adjustment.

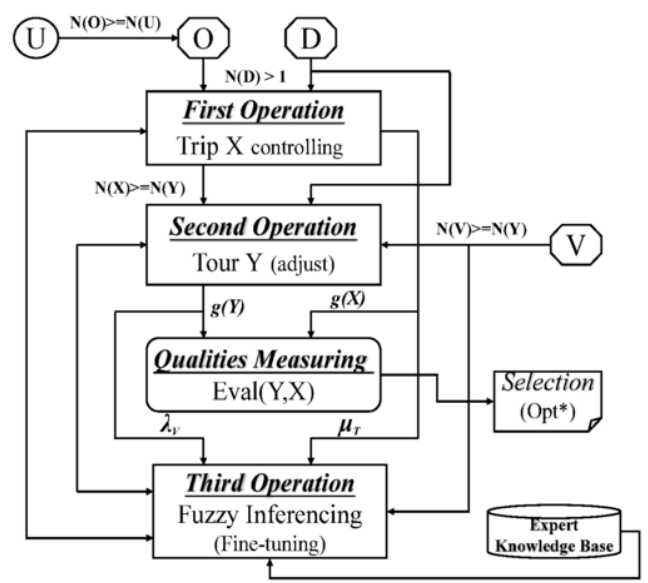

Figure 11. Control algorithm with HIMS+ Model.

Based on the above operations, the optimization about the VRSDP/MD problem can be completed. 


\section{The Evaluations for Experiments}

The fourth part proves the validity and convergence of HIMS+ model through experiments. And HIMS+ is a complex model, so it is difficult to verify the characteristics of the model analytically. Components of the HIMS+ model are applied to the actual data sets from real oil companies, with an average of 40 to 60 orders per day delivered by 24 tankers at 3 depots, in 2 types (14kl and 20kl) to save operating costs. The transportation network in the metropolis of Japan (Tokyo area) is also presented in the form of digital map, and the transportation cost information is calculated by Dijkstra algorithm [11].

In this experiment, two experiments with 3-day order data are performed. According to the consequence of the experimental, The HIMS+ model evaluates the efficiency and flexibility of VRSDP/MD problems from multiple perspectives.

\subsection{Experiment A: Working with All Vehicles.}

Table 3. The Initial Parameter in Experiment A.

\begin{tabular}{ccccc}
\hline RWt & $\mathbf{E W t}$ & $\boldsymbol{\mu}_{\mathrm{T}}$ & $\boldsymbol{\lambda}_{\mathbf{V}}$ & $\boldsymbol{\rho}_{\mathbf{i}}(\mathbf{i}=\mathbf{1} \sim \mathbf{6})$ \\
\hline $6 \mathrm{~h}$ & $2 \mathrm{~h}$ & {$[0.45,0.55]$} & {$[0.45,0.55]$} & $300,100,100,0,30,30$ \\
\hline
\end{tabular}

Table 3 introduce the original parameter in experiment A. Within the safety zone, the trip balance and the vehicle balance are set to fuzzy measure from 0.45 to 0.55 .

The total running cost is mainly concerned as $\rho_{1}=300$. The objective of Experiment $\mathrm{A}$ is to develop the best plan for all vehicular trip jobs, including the best routes, while maintaining the optimizing balance in the overall evaluation standards. Table 4 describes the explicit Execution consequence of Experiment A.

Table 4. Execution consequence of Experiment A.

\begin{tabular}{|c|c|c|c|c|c|c|c|}
\hline date & Order (\#) & $\mathbf{T}_{\text {cost }}^{[m]}(\min )$ & $\mathrm{C}_{\text {cost }}^{[n]}(\%)$ & $\mathbf{B}_{\text {cost }}^{[n]}(\min )$ & $S_{T}^{[0]}(\%)$ & $S_{v}^{[n]}(\%)$ & (a) Best \\
\hline $12 / 09$ & 40 & 9608 & 90.56 & 88.44 & 95.37 & 95.27 & $86^{\text {th }}$ \\
\hline $12 / 11$ & 48 & 10681 & 92.38 & 55.21 & 91.45 & 96.93 & $128^{\text {th }}$ \\
\hline $12 / 14$ & 42 & 9674 & 94.02 & 72.10 & 91.87 & 95.92 & $136^{\text {th }}$ \\
\hline
\end{tabular}

4.2. Experiment B: Working with Minimum Vehicles.

Table 5. The Initial Parameter in Experiment B.

\begin{tabular}{ccccc}
\hline RWt & $\mathbf{E W t}$ & $\boldsymbol{\mu}_{\mathrm{T}}$ & $\boldsymbol{\lambda}_{\mathbf{V}}$ & $\boldsymbol{\rho}_{\mathbf{i}}(\mathbf{i}=\mathbf{1} \sim \mathbf{6})$ \\
\hline \multirow{2}{*}{$8 \mathrm{~h}$} & $4 \mathrm{~h}$ & {$[0.5,0.6]$} & {$[0.5,0.6]$} & $300,200,200,100,30,30$ \\
\hline
\end{tabular}


Table 6. Execution consequence of Experiment B.

\begin{tabular}{|c|c|c|c|c|c|c|c|c|}
\hline date & $\begin{array}{c}\text { Order } \\
(\#) \\
\end{array}$ & $T_{\text {cost }}^{|r|}(\min )$ & $\mathrm{C}_{\text {cost }}^{\prime \prime 1)}(\%)$ & $\begin{array}{r}\mathbf{B}_{\text {cost }}^{r+1} \\
(\mathbf{m i n})\end{array}$ & $\begin{array}{l}V_{\text {cost }}^{\text {lit }} \\
\text { (unit) }\end{array}$ & $\mathbf{S}_{\mathrm{T}}^{(\mathrm{rl})}(\mathbf{\%})$ & $\mathrm{S}_{\mathrm{v}}^{\mathrm{rl}}(\mathbf{\%})$ & (b) Best \\
\hline $12 / 09$ & 40 & 9587 & 91.96 & 53.72 & 6 & 92.37 & 87.98 & $167^{\text {th }}$ \\
\hline $12 / 11$ & 48 & 10024 & 91.61 & 49.78 & 6 & 94.08 & 84.17 & $184^{\text {th }}$ \\
\hline $12 / 14$ & 42 & 8910 & 94.67 & 57.73 & 8 & 92.85 & 84.13 & $85^{\text {th }}$ \\
\hline
\end{tabular}

Table 5 introduce the original parameter in experiment B. The safety zones are both shifted to $[0.5,0.6]$ to allow for slight overwork too much. $\mathrm{T}_{\text {cost }}, \mathrm{C}_{\text {cost }}$, and $\mathrm{B}_{\text {cost }}$ are mainly concerned. The objective of Experiment B is to develop an optimal plan for a trip work with only a few vehicles (including optimal routes) while maintaining the optimal balance of all evaluation criteria. This situation is often due to regular maintenance of the vehicle, inspection, etc., as well as the driver's holiday. Table 6 shows the detailed execution consequence of experiment B.

\subsection{Experimental Results and analysis}

In this subsection, Detailed analysis of the calculation process is recorded in the database, and the superiority of HIMS+ model is discussed, such as the consequence of experimental, algorithms and some applications in system.

\subsubsection{The Results and Discussion of Experimental}

The experiment A and B cover almost all the major situations, such as all the work when the demand is high, and periodic inspection, maintenance and other part of the work are considered.

Table 7 compares the data of the HIMS+ model with that of an experienced operator scheme. In the items of $\mathrm{S}_{\mathrm{T}}, \mathrm{S}_{\mathrm{V}}, \mathrm{T}_{\mathrm{cost}}$, and $\mathrm{V}_{\text {cost }}$, the computational power of HIMS+ model is superior than human experts.

Table 7. Data compare with HIMS+ and Expert.

\begin{tabular}{ccccccc}
\hline Operator & $\begin{array}{c}\text { Working } \\
\text { Cost }\end{array}$ & $\begin{array}{c}\text { Loading } \\
\text { Rate }\end{array}$ & $\begin{array}{c}\text { Working } \\
\text { Balance }\end{array}$ & $\begin{array}{c}\text { Working } \\
\text { Capacity }\end{array}$ & $\begin{array}{c}\text { Fuzzy } \\
\text { measures } \\
\text { one }\end{array}$ & $\begin{array}{c}\text { Fuzzy } \\
\text { measures } \\
\text { two }\end{array}$ \\
\hline Expert & good & $\leqq 90 \%$ & $\leqq 90 \mathrm{~min}$ & good & $\geqq 80 \%$ & $\geqq 80 \%$ \\
HIMS+_E1 & better & $\geqq 90 \%$ & $\leqq 90 \mathrm{~min}$ & better & $\geqq 91 \%$ & $\geqq 95 \%$ \\
HIMS+_E2 & better & $\geqq 91 \%$ & $\leqq 60 \mathrm{~min}$ & better & $\geqq 92 \%$ & $\geqq 84 \%$
\end{tabular}

\subsubsection{Probing Into Algorithms}

The System of traditional methods or some expert process transportation problems with sub-problems about VRSDP/MD problems are considered individually. The HIMS+ model integrates these sub-problems into three layers that are tightly connected in an integrated manner. Table 8 shows the differences by comparing the three computing methods. Because the HIMS+ model deals with the VRSDP/MD problem in the integrated approach, a better balance of trips, tours and vehicles can be achieved. 
Table 8. The comparison of the three computing methods.

\begin{tabular}{ccccc}
\hline Algorithm & Process & Data Type & Data Architecture & Optimum Means \\
\hline Conventional & Individual & Crisp $\{0,1\}$ & Linear & Meta or Inter \\
Expert & Individual & Crisp $\{0,1\}$ & ------ & Programming \\
HIMS + & Synthesis & Fuzzy $[0,1]$ & Object-oriented & Meta + Fuzzy \\
& & &
\end{tabular}

\subsubsection{Extension of System Applications.}

One interesting perspective is how the HIMS+ model can be used for components in system applications. Table 9 expresses the superiority of the HIMS+ model from the perspective of practical application of the system. The results shows that HIMS+ model has simple inputting parameters, multiple evaluation objectives, strong flexibility, and can adjust the balance better. In practical application, the used of cab is also very fast.

Table 9. The comparison of the two systems.

\begin{tabular}{cccccc}
\hline Application & $\begin{array}{c}\text { Input } \\
\text { Parameters }\end{array}$ & $\begin{array}{c}\text { Objective } \\
\text { Functions }\end{array}$ & $\begin{array}{c}\text { Objectives } \\
\text { Balance }\end{array}$ & $\begin{array}{c}\text { Process } \\
\text { Time }\end{array}$ & Flexibility \\
\hline HIMS+ & Fewer & Many & Better & Faster & Better \\
Conventional & Many & Few & Bad & Slow & Bad \\
\hline
\end{tabular}

\section{Conclusion}

A new concept of VRSDP/MD problem in real world is proposed and formalized by fuzzy set theory. The expanded computing model including hierarchical multiplex structure, named the HIMS+ model, and an operation strategy is proposed that based on heuristic optimization algorithm and fuzzy reasoning. The HIMS+ model is implemented using the object-oriented multiple paradigms as software components. The algorithm of optimization is based on meta-programming and fuzzy programming.

Two experiments carry out with the data set from the car distribution centre to make the optimizing dispatching scheduling for all vehicles working while maintaining the quasi-optimization balance of all evaluative standard. There, twenty-four tank lorries with two kinds $(20 \mathrm{kl} \& 14 \mathrm{kl})$ are delivered on daily and running costs are reduced by using three warehouse points.

The experimental results show that the consequence of HIMS + model test is higher than those of experience dispatcher in six evaluation indexes. Because the HIMS+ model and its algorithms are based on three layers (First layers, Second Layers, and Third layers) containing object-oriented technology, heuristics, and fuzzy inference, the HIMS + model can find effective solutions with intelligence and flexibility like expert dispatchers. Because there are few important inputting parameters \& the computing core engine is packaged as the software modules, the HIMS + model is a functional system application for real-world problems of VRSDP/MD. The evaluation and analysis of the experimental results show that HIMS+ is a reliable, high speed and high 
efficiency component with contrasts of expert system or traditional methods. Since the problem of VRSDP/MD is a NP-hard problem with wide applications, they are difficult to prove the astringency of HIMS+ computing model through mathematical means analysis. However, the experimental calculation shows that HIMS+ model makes the mass of the solution increase monotonically to the upper bound as a function of operation time. By analysis and inference to the process information recorded in the experiment, the robustness of the HIMS+ model in practical application is further verified.

The HIMS+ model is the basis of some problems like CSP (Combinatorial Optimization Problem) or COP (Constraint Satisfaction Problem). The HIMS+ model will be applied to solve the practical transportation problems, including daily land, weekly air cargo or monthly shipping and so on.

\section{Reference}

[1] Barcelo J, Grzybowska H and Pardo S October 2007 Vehicle routing and scheduling models, simulation and city logistics

[2] Laporte G and Osman I, 1995 Routing problems: A bibliography, Ann. Oper. Res., vol 61 pp 227-62

[3] Afifi S, Dang D and Moukrim A August 2013 A Simulated Annealing Algorithm for the Vehicle Routing Problem with Time Windows and Synchronization Constraints $7^{\text {th }}$ International Conference, Learning and Intelligent Optimization (LION7) (Catania, Italy). pp 259-65

[4] Osman I 1993 Metastrategy simulated annealing and tabu search algorithms for the vehicle routing problem, Ann. Oper. Res. vol 41 pp 421-51

[5] Cheeneebash J and Nadal C August 2010 Using Tabu Search Heuristics in Solving the Vehicle Routing Problem with Time Windows: Application to a Mauritian Firm.

[6] Dorner K and Schilde M February 2017 The Tabu Search Heuristic for the Vehicle Routing Problem with Soft Time Windows (Johannes Kepler Universitat Linz)

[7] Benyahia I and Potvin J 1998 Decision support for vehicle dispatching using genetic programming. IEEE Tran. Sys. M. Cyber., Part a, System and Humans, vol 28 No 3 pp 306-14

[8] Chen K, Dong F and Hirota K August 2020 A Calculation Model of Hierarchical Multiplex Structure with Fuzzy Inference for VRSD Problem, Recent Development in Fuzzy logic and fuzzy sets, Studies in Fuzziness and Soft Computing (Springer), Vol 391 pp175-99

[9] Glover F, Taillard E and Werra D 1993 A user's guide to tabu search, Annals of Operations Research, vol 41 pp 3-28

[10] Gallo C and Capozzi V July 2019 A Simulated Annealing Algorithm for Scheduling Problems, Journal of Applied Mathematics and Physics pp 2579-94

[11] Dijkstra E 1959 A note on two problems in connection with graphs Numerische Mathematik, vol 1 pp 269-71

[12] Meyer B March 2000 Object-Oriented Software Construction second edition ISE Inc. Santa Barbara (California) 